\title{
The Association of Vitamin D with MicroRNA in Preeclampsia
}

\author{
Xingli Deng ${ }^{1}$ and Yuan Qian*2,3 \\ ${ }^{1}$ Department of Neurosurgery, $1^{\text {st }}$ Affiliate Hospital of Kunming Medical University, China \\ ${ }^{2}$ Department of Laboratory Medicine, $1^{\text {st }}$ Affiliated Hospital of Kunming Medical University, China \\ ${ }^{3}$ Genetic Diagnosis Center, Women and Children Hospital, China
}

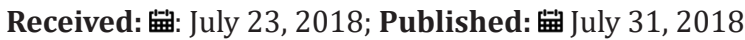

*Corresponding author: Yuan Qian, The Key Laboratory of Laboratory Medicine of Yunnan Province \& Prenatal Diagnosis Lab, Department of Laboratory Medicine, $1^{\text {st }}$ Affiliated Hospital of Kunming Medical University. Genetic Diagnosis Center, Women and Children Hospital, Kunming, Yunnan, China

\section{Mini Review}

It has been extensively investigated that the association between vitamin D deficiency and adverse outcomes in diseases with pregnancy, including preeclampsia. The dysregulation of microRNA expression in placenta with preeclampsia had also been reported in many studies. However, the association of vitamin D deficiency and dysregulation of microRNA in the placenta had been rarely reported. In our manuscript, we aim to shortly review the existing literature regarding the vitamin D deficiency during pregnancy, the factors associated with vitamin D deficiency, and the association of vitamin D with microRNA in preeclampsia.

\section{Vitamin D deficiency during Pregnancy}

Vitamin D metabolism during pregnancy has striking differences compared to the non-pregnant state .1,25-dihidroxyivitamin $\mathrm{D}(1,25(\mathrm{OH}) 2 \mathrm{D})$, the active form of vitamin $\mathrm{D}$ has been converted from $25-0 H$ at the renal site in the non-pregnant state. In the pregnancy, the placenta is thought to be the major site of vitamin D metabolism, providing $1,25(\mathrm{OH}) 2 \mathrm{D}$ in vitro [1]. Vitamin D has an important role not only in maintaining an adequate level of minerals in bone mineralization but also acting as a potent immunomodulator in immunoregulating effect [2,3]. Vitamin D receptors are broadly present on nearly all immune cells, like Th1, Th2, Tregs, Th17, monocytes, B-lymphocytes and microphage $[4,5]$. The 1, 25(OH) 2D could modulate the expression of cytokines by bounding to its receptor. Observational studies supported the significant association between lower vitamin D concentrations and higher risk of preeclampsia. Indeed, studies supported that lower 25-OH vitamin D serum levels and lower 1,25(OH)2 vitamin D levels in patients with preeclampsia compared to healthy pregnant women with the same gestational age [6,7]. However, there are still some observational studies found no difference in vitamin $\mathrm{D}$ concentrations in women with preeclampsia compared to normotensive women [8]. Andraž Dovnik think that the contradiction could be contributed by their variable definition of preeclampsia, discrepancies in the analysis of confounding factors and differences in the gestational age at which vitamin D concentration was assessed, or a low number of patients with preeclampsia included in the study [9]. Vitamin D status is influenced by the gestational age, obesity and ethnicity. These factors can also affect the risk of preeclampsia as well. Till now, the mechanism of vitamin D deficiency in patients with preeclampsia has not fully understood.

\section{MicroRNA in the Placenta with Preeclampsia}

MicroRNAs (miRNAs) are short non-coding RNAs. Dysregulation of miRNAs is implicated in the pathogenesis of preeclampsia, including placental specific microRNAs and differentially expressed microRNAs $[10,11]$. The levels of miRNAs expression are influenced by several physiological factors, like race, different type of preeclampsia. The placenta-specific miRNAs are mainly clustered in C14MC, C19MC and miR-371-373cluster. Altered expression of 7 miRNAs, including miR-210, miR-30a-3p, miR-518b, miR-524, miR-17-3p, miR-151, and miR-193b had been reported in the placental tissues with PE in Chinese [12]. Lykoudi A et al reported that miR-431, miR-518a-5p and miR-124 were over-expressed in early onset PE (EOPE) complicated placentas as compared to controls, whereas miR-544 and miR-3942 were down-regulated in EOPE [13]. However, miRNAs with differential expression in different study showed a minimal overlapping pattern, even contradictory data were also reported. Shortcomings associated with placental sampling, the onset time of PE in the different studies were considered the main factors contributing the phenomena. Abnormal microRNA expression may have a role in the development of preeclampsia as it affects the proliferation, migration, and invasion of the trophoblast cells, spiral artery remodeling, and angiogenesis.

\section{Vitamin D and miRNA in Preeclampsia}

vitamin D and miRNAs might play an important role in the pathogenesis of PE. However, the association between the serum 
level of vitamin D and miRNAs expression involved in PE patients has not been reported. Results from other study have shown that 1,25-didroxyvitamin D status was associated with the expression of miRNAs in patients with coronary artery diseases [14]. In our study, 42 microRNAs with differential expression in PE placenta in lack of vitamin D were found, and the association between vitamin D, miRNAs expression and preeclampsia were analyzed (under review). Further studies are required to investigate whether differential microRNA which might be associated with vitamin D receptor and vitamin D signaling pathway through the function predication and analysis with Target Scan database. These results may provide the data to investigate vitamin D supplementation, which would reduce the risk of severe preeclampsia and improve pregnancy outcomes.

\section{References}

1. Karras SN, Wagner CL, Castracane VD (2017) Understanding vitamin D metabolism in pregnancy: From physiology to pathophysiology and clinical outcomes. Metabolism Oct 21.

2. Kumar A, Singh MP, Kumar RS, Ratho RK (2018) 25-Hydroxyvitamin D3 and 1,25 Dihydroxyvitamin D3 as an Antiviral and Immunomodulator Against Herpes Simplex Virus-1 Infection in HeLa Cells. Viral Immunol May 24.

3. Lee RU, Won SH, Hansen C, Crum Cianflone NF (2018) 25-hydroxyvitamin $D$, influenza vaccine response and healthcare encounters among a young adult population. PLoS One. Feb 9 13(2): e0192479.

4. Rimmelzwaan LM, van Schoor NM, Lips P, Berendse HW, Eekhoff EM (2016) Systematic review of the relationship between vitamin D and Parkinson's disease. J Parkinsons Dis 6: 29-37.

\section{ISSN: 2574-1241}

DOI: 10.26717/BJSTR.2018.07.001508

Yuan Qian. Biomed J Sci \& Tech Res

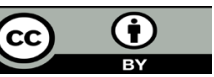

This work is licensed under Creative Commons Attribution 4.0 License

Submission Link: https://biomedres.us/submit-manuscript.php
5. Ernst JB, Zittermann A, Pilz S, Kleber ME, Scharnagl H, et al. (2017) Independent associations of vitamin $\mathrm{D}$ metabolites with anemia in patients referred to coronary angiography: the LURIC study. Eur J Nutr 56: 1017-1024.

6. Hyppönen E, Cavadino A, Williams D, Fraser A, Vereczkey A, et al. (2013) Vitamin $\mathrm{D}$ and pre-eclampsia: original data, systematic review and meta-analysis. Ann Nutr Metab 63(4): 331-340.

7. Thorne Lyman A, Fawzi WW (2012) Vitamin D during pregnancy and maternal, neonatal and infant health outcomes: a systematic review and meta-analysis. Paediatr Perinat Epidemiol Jul 26 Suppl 1: 75-90.

8. Shand AW, Nassar N, Von Dadelszen P, Innis SM, Green TJ (2010) Maternal vitamin D status in pregnancy and adverse pregnancy outcomes in a group at high risk for pre-eclampsia. BJOG Dec 117(13): 1593-1598.

9. Dovnik A, Mujezinović F (2018) The Association of Vitamin D Levels with Common Pregnancy Complications. Nutrients 10(7).

10. Morales Prieto DM, Ospina Prieto S, Chaiwangyen W, Schoenleben M, Markert UR (2013) Pregnancy-associated miRNA-clusters. J Reprod Immunol Mar 97(1): 51-61.

11. Pineles BL, Romero R, Montenegro D, Tarca AL, Han YM, et al. (2007) Distinct subsets of microRNAs are expressed differentially in the human placentas of patients with preeclampsia. Am J Obstet Gynecol Mar 196(3): 261.

12. Xu P, Zhao Y, Liu M, Wang Y, Wang H, et al. (2014) Variations of microRNAs in human placentas and plasma from preeclamptic pregnancy. Hypertension Jun 63(6): 1276-1284.

13. Lykoudi A, Kolialexi A, Lambrou GI, Braoudaki M, Siristatidis C, et al. (2018) Dysregulated placental microRNAs in Early and Late onset Preeclampsia. Placenta Jan 61: 24-32.

14. Sheane BJ, Smyth P, Scott K, Aziz R, Buckley M, et al. (2015) An Association between MicroRNA-21 Expression and Vitamin D Deficiency in Coronary Artery Disease. Microrna 4(1): 57-63.

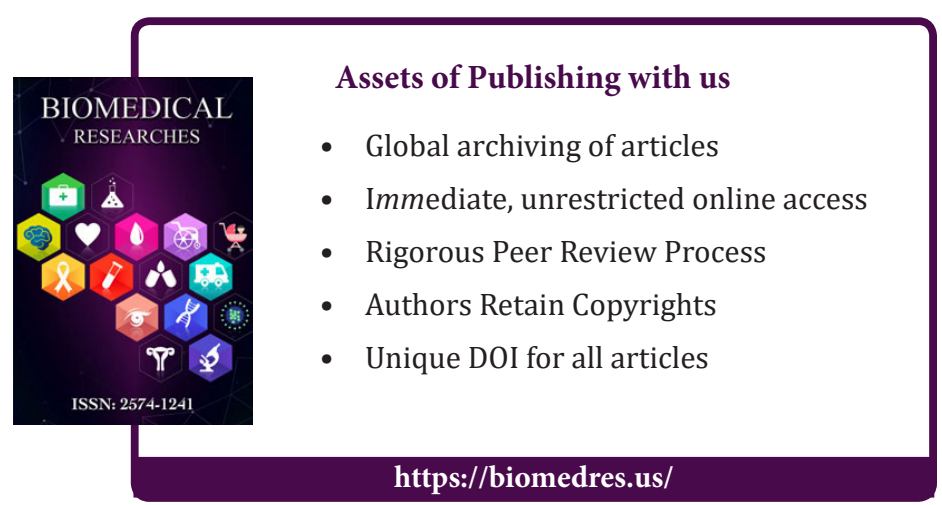

